\title{
Points saillants de la 7e édition des Normes canadiennes pour la lutte antituberculeuse
}

\author{
Gale Rowe M. ${ }^{{ }^{\star}}$, Menzies $\mathrm{D}^{2}{ }^{2}$, Sutherland J. ${ }^{3}$ et Wong $\mathrm{T}^{1}$ \\ au nom des réviseurs et des auteurs des chapitres
}

\author{
${ }^{1}$ Agence de la santé publique du Canada, Ottawa (Ontario) \\ ${ }^{2}$ Université McGill, Montréal (Québec) \\ ${ }^{3}$ Société canadienne de thoracologie - Association pulmonaire du Canada, Ottawa (Ontario) \\ * Auteure-ressource : Margaret.galerowe@phac-aspc.gc.ca
}

\section{Introduction}

Au Canada, la tuberculose demeure un problème de santé important. L'incidence générale signalée de cas de tuberculose active continue de baisser, mais certaines sous-populations, comme les Autochtones et les personnes nées à l'étranger, sont touchées de façon disproportionnée. À ce jour, la recherche spécialisée nous permet de progresser dans notre compréhension de la pathogenèse, de l'immunologie et de l'épidémiologie de la tuberculose, ainsi que dans l'élaboration de nouveaux outils thérapeutiques et de diagnostic. Cette $7^{\mathrm{e}}$ édition des Normes canadiennes pour la lutte antituberculeuse (les Normes) a été minutieusement révisée afin d'intégrer ces nouveaux renseignements, en s'appuyant sur les six versions précédentes(1). Chaque chapitre est écrit par des auteurs originaires d'un peu partout au Canada possédant une expertise dans des domaines précis. Les Normes ont pour vocation de fournir des renseignements sur les meilleures pratiques et non pas de remplacer les consultations avec les professionnels de la santé. Elles ne remplacent pas les exigences juridiques, réglementaires, politiques et en matière de pratiques des provinces/territoires, ni les lignes directrices professionnelles qui régissent la pratique des professionnels de la santé dans leur compétence respective, et dont les recommandations peuvent différer en raison de l'épidémiologie ou du contexte sur place. Le présent résumé met en lumière certains des principaux changements dans les recommandations.

\section{Approche}

Les Normes ont été co-élaborées par la Société canadienne de thoracologie, l'Association pulmonaire du Canada et l'Agence de la santé publique du Canada, en collaboration avec l'Association pour la microbiologie médicale et l'infectiologie Canada. Tout comme les éditions précédentes, la $7^{e}$ édition des Normes repose sur les meilleures preuves scientifiques disponibles. Chaque chapitre a été rédigé par un ou plusieurs auteurs ayant une expertise en matière de prévention et de contrôle de la tuberculose. Ces auteurs ont examiné avec attention toutes les preuves publiées, en particulier les études les plus récentes, et les ont résumées et évaluées à l'aide de la méthodologie GRADE (Grading of Recommendations, Assessment, Development and Evaluation). Les recommandations sont considérées comme étant fortes ou conditionnelles en fonction des avantages, des risques et du fardeau potentiels.

\section{Résultats}

Les Normes comprennent des mises à jour importantes pour les meilleures pratiques en matière de prévention et de contrôle de la tuberculose (Tableau 1). Cela inclut, notamment, une nouvelle approche pour la collecte des expectorations, afin que les échantillons de cas suspectés de tuberculose puissent tous être prélevés le même jour, ce qui permettrait de réduire le taux d'abandon et d'améliorer le rendement. On y trouve également un nouveau test d'amplification des acides nucléiques contenu dans une cartouche pour détecter $M$. tuberculosis. Les tests disponibles concernant la réaction de polymérisation en chaîne en temps réel permettent de détecter $M$. tuberculosis et la résistance en deux heures. Les résultats doivent être confirmés par des frottis et des cultures systématiques. Il existe de nouvelles recommandations sur l'utilisation appropriée du test cutané à la tuberculine et des tests de libération d'interféron-gamma, même lorsqu'aucun des tests n'est indiqué. 
Des changements majeurs ont été apportés aux recommandations concernant le schéma et la durée thérapeutiques pour traiter la tuberculose, l'infection tuberculeuse latente, la tuberculose pharmacorésistante et la tuberculose infantile. Plusieurs recommandations en matière de pratique sont proposées pour la tuberculose pharmacorésistante. Tout comme on a souligné la nécessité d'un dépistage systématique du VIH chez tous les patients atteints de tuberculose, le dépistage systématique de l'infection tuberculeuse latente est préconisé chez tous les patients infectés par le $\mathrm{VIH}$. En raison d'importantes interactions médicamenteuses possibles, les personnes souffrant d'une co-infection tuberculose-VIH devraient être prises en charge par un médecin expert dans les soins de cette co-infection ou, du moins, en étroite collaboration avec lui. Dans le cadre des soins de santé, on a apporté des changements majeurs aux recommandations pour les tests de base des travailleurs de la santé, ainsi que de nouveaux renseignements sur le risque de transmission. Selon les changements apportés aux recommandations concernant l'utilisation du vaccin bacille de Calmette-Guérin (BCG), ce vaccin n'est plus recommandé pour certains groupes ou seulement utilisé à titre exceptionnel dans d'autres groupes. Pour ce qui est du suivi des contacts, des détails sont fournis sur le passage du modèle classique à une priorisation des contacts en fonction des caractéristiques du cas-source et de la réceptivité de la personne exposée.

\section{Tableau 1 : Faits saillants des changements clés apportés à la $7^{\mathrm{e}}$ édition des Normes canadiennes pour la lutte antituberculeuse}

\begin{tabular}{ll}
\hline Chapitre \\
\hline 1. Épidémiologie \\
\hline 2. $\begin{array}{l}\text { Pathogenèse et } \\
\text { transmission de la } \\
\text { tuberculose }\end{array}$ \\
\hline 3. $\begin{array}{l}\text { Diagnostic de la } \\
\text { tuberculose active et de } \\
\text { la pharmacorésistance }\end{array}$
\end{tabular}

Faits saillants et nouvelles recommandations

Données de surveillance fournies jusqu'en 2010.

Description de la probabilité de transmission et de progression pour des souspopulations précises.

Nouvelle approche de collecte des expectorations composée de trois échantillons recueillis le même jour, avec au plus une heure entre chaque prélèvement. Description de l'utilisation de l'outil de diagnostic qu'est le test d'amplification des acides nucléiques contenu dans une cartouche. Les tests disponibles concernant la réaction de polymérisation en chaîne en temps réel permettent de détecter $M$. tuberculosis et la résistance en deux heures.

4. Diagnostic de l'infection tuberculeuse latente

5. Traitement de la tuberculose

6. Traitement de l'infection tuberculeuse latente

\section{Tuberculose non respiratoire}

8. Tuberculose pharmacorésistante

9. Tuberculose infantile

10. Tuberculose et virus de l'immunodéficience
Nouvelles recommandations concernant le test cutané à la tuberculine et les tests de libération d'interféron-gamma.

Changements majeurs apportés aux recommandations concernant le schéma et la durée thérapeutiques. Renseignements sur les doses pour les médicaments de première et de deuxième intention, d'autres traitements intermittents de rechange, et un traitement sur mesure pour des sous-populations précises.

Changements majeurs apportés aux recommandations concernant le choix d'un traitement pour une infection tuberculeuse latente, avec des recommandations pour de nouveaux schémas plus courts, ainsi que le traitement des contacts des cas pharmacorésistants.

Nouveaux renseignements sur la sensibilité et la particularité des tests diagnostiques pour les différentes formes de tuberculose extrapulmonaire et l'utilisation de stéroïdes comme traitement d'appoint.

Recommandation des changements majeurs à apporter au traitement et présentation de moyens de réduire la pharmacorésistance.

Nouveaux renseignements sur les groupes à risque et changements majeurs apportés au diagnostic et au traitement.

Changements majeurs apportés aux recommandations en matière de traitement de l'infection tuberculeuse latente et du VIH, ainsi que concernant le traitement de la tuberculose chez les personnes nécessitant un traitement antirétroviral. Insistance 


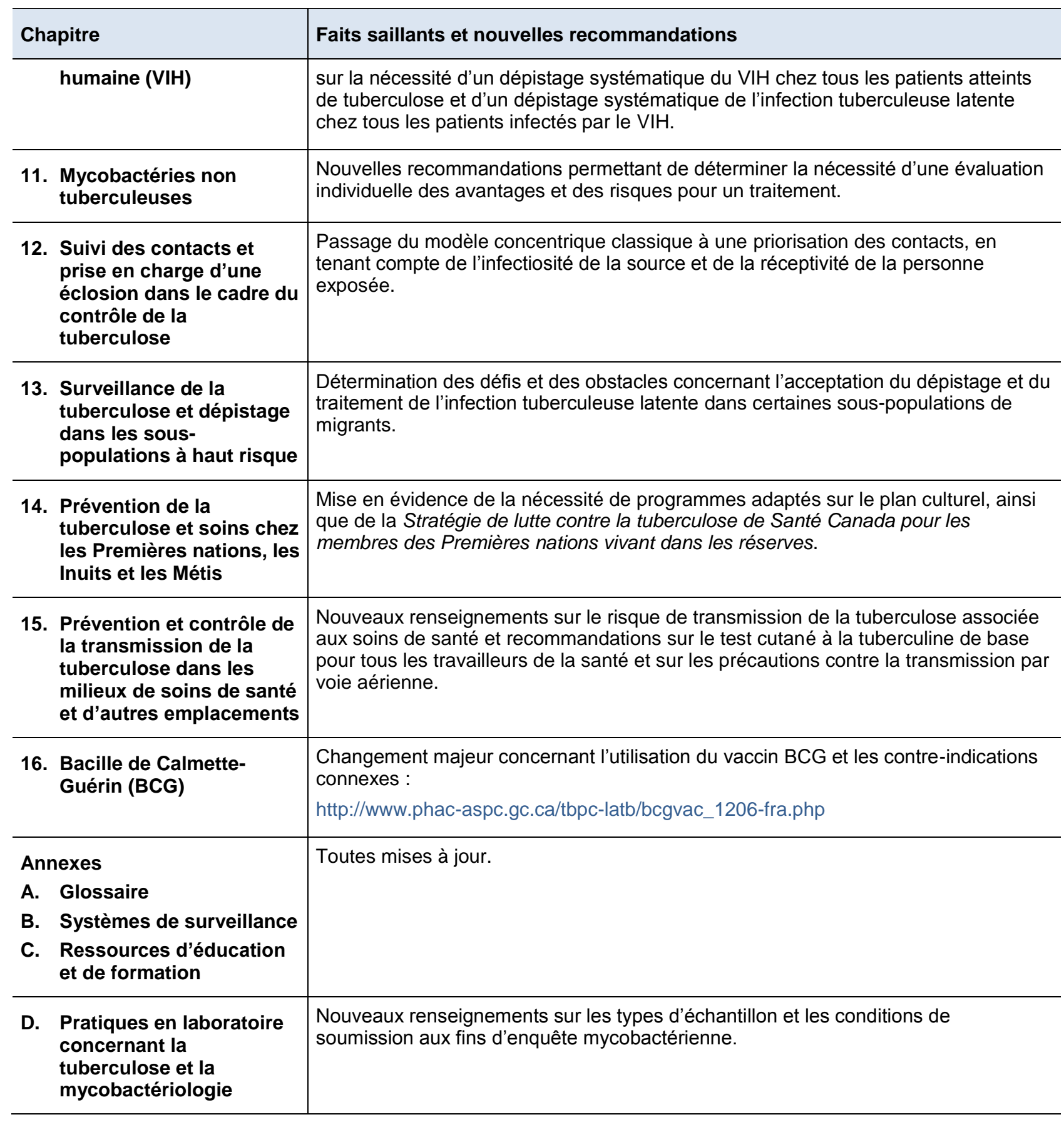

\section{Conclusions}

Les Normes constituent la base de la prévention et du contrôle de la tuberculose au Canada. La $7^{\mathrm{e}}$ édition met en lumière la contribution de nouveaux outils diagnostiques et thérapeutiques pour le diagnostic précoce et la prise en charge réussie des cas, dans le contexte de nouveaux problèmes liés à la résistance aux antimicrobiens et à la co-infection. 


\section{Références}

(1) Menzies D. et Wong T. (éd.) 2013. Normes canadiennes pour la lutte antituberculeuse, $7^{\mathrm{e}}$ édition. Société canadienne de thoracologie, Association pulmonaire du Canada, Agence de la santé publique du Canada. Accès : http://www.lignesdirectricesrespiratoires.ca/normes-antituberculeuse-2013

\section{Remerciements}

Mille mercis au réviseur Dick Menzies ainsi qu'aux coréviseurs Edward Ellis, Richard Long, Madhukar Pai et Thomas Wong.

Également, mille mercis aux auteurs des chapitres :

Alvarez G. G., Archibald C. P., Avendano M., Behr M., Christianson S., Cook V., Demers A.-M., Ellis E., Elwood K., Embil J., Fisher D., Gallant V., Greenaway C., Halverson J., Houston S., Jamieson F., Jarand J., Khan K., Kitai I., Kunimoto D., Long R., Marras T. K., Menzies D., Minion J., Ogunremi T., Orr P., Pai M., Rea E., Rivest P., Schwartzman K., Sharma M., Wobeser W. L., Wolfe J. et Wong, T.

\section{Conflit d'intérêts}

II n'y a aucun conflit d'intérêts à déclarer.

\section{Financement}

Ce travail a été appuyé par l'Agence de la santé publique du Canada. 